\title{
Influência da orientação religiosa na qualidade de vida de idosos \\ ativos
}

\author{
Influence of religascrientaionin thequlity of lifeof ativeddely
}

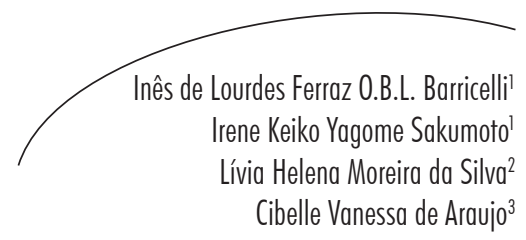

Resumo

Objetivo: Identificar modalidades de orientação religiosa, se intrínseca ou extrínseca, e possíveis relações com a qualidade de vida de idosos ativos, além de compreender as manifestações do fenômeno religioso para uma adequada atuação clínica nos desafios das práticas do atendimento ao idoso. Métodos: A amostra foi composta de 60 idosos ativos, frequentadores de grupos de convivência na cidade de São José dos Campos, SP. Foram aplicados dois instrumentos: questionário genérico Medical Outcomes Study 36-Item Short-Form Health Survey (SF-36), para avaliar a qualidade de vida, e Escala de Orientação Religiosa Intrínseca e Extrínseca, além de entrevista orientada por um questionário sociodemográfico, elaborado pelas pesquisadoras. Os dados foram tratados estatisticamente utilizando o teste $\mathrm{t}$ Student bilateral, a um nível de confiança de 5\%. Resultados: Foram avaliados 11 homens (18\%) e 49 mulheres (82\%), com idade entre 61 e 85 anos. Não houve diferença estatisticamente significativa em todos os domínios relacionados do SF-36 entre as mulheres com religiosidade intrínseca e extrínseca. Entre os homens, somente foi encontrada diferença estatisticamente significativa no domínio "estado geral de saúde", que foi considerado melhor entre aqueles com religiosidade intrínseca. Conclusão: De modo geral, não se verificaram relações significativas entre a orientação religiosa e a qualidade de vida. Pode-se aventar, diante disso, que esses resultados se devem ao fato de a população estudada participar de grupos de convivência e morar em cidade com alto nível de futuridade, sentindo-se amparada nos aspectos de proteção, participação e saúde.

\section{Abstract}

Objective: Identify forms of religious orientation, if intrinsic or extrinsic, and possible relations to the quality of life for active seniors and to understand the manifestations of the religious phenomenon for adequate clinical practices on the challenges of senior

\footnotetext{
Curso de Pós-Graduação em Gerontologia. Centro de Estudos Firval. São José dos Campos, SP, Brasil.

2 Curso de Pós-Graduação em Engenharia Biomédica. Universidade Camilo Castelo Branco. São José dos Campos, SP, Brasil.

3 Curso de Fisioterapia. Departamento de Medicina Translacional. Universidade Federal de São Paulo. São Paulo, SP, Brasil.
}

\section{Palavras-chave}

Envelhecimento. Qualidade de vida. Religião. Idoso. Centros de Convivência e Lazer. São José dos Campos, SP. 
care. Methods: The sample comprised 60 active elderly, attending supporting groups, in the city of São José dos Campos, SP. We used the instruments: generic questionnaire Medical Outcomes Study 36-Item Short-Form Health Survey (SF-36), to assess the quality of life, and the Intrinsic-Extrinsic Religious Orientation Scale, as well as an interview guided by a socio-demographic questionnaire, developed by the researchers. Data were statistically treated using the bilateral Student t-test, to a confidence level of 5\%. Results: We evaluated 11 men (18\%) and 49 women (82\%), aged 61-85 years. There was no statistically significant difference in all areas of the SF-36 among women with intrinsic and extrinsic religiosity. Only statistically significant difference was found among men in the field "General Health", which was considered better among those with intrinsic religious. Conclusion: Overall, there were no significant relationships between religious orientation and quality of life. This may suggest that these results are due to the fact that the study population participates in support groups, and live in a city with high level of futurity, feeling supported in the aspects of protection, participation and health.
Key words: Aging. Quality of life. Religion. Aging. Centers for Living and Leisure. Sao Jose dos Campos, SP.

\section{INTRODUÇÃO}

Com a elevação da expectativa de vida da população, há um maior interesse, por parte dos profissionais das diversas áreas da saúde, em estudos e iniciativas que auxiliem na obtenção e/ ou manutenção da independência, autonomia e qualidade de vida dos indivíduos idosos. ${ }^{1}$

O termo "qualidade de vida" não deve ser confundido com estilo, condição, situação de vida e/ou estado de saúde. ${ }^{2}$ A qualidade de vida é a percepção do indivíduo de sua posição na vida, no contexto da cultura e dos sistemas de valores nos quais ele vive em relação a seus objetivos, expectativas, padrões e preocupações. ${ }^{3}$

Para que o processo de envelhecimento venha acompanhado de qualidade de vida, deve-se dar oportunidade contínua ao idoso de participação ao longo da vida, respeitandose suas necessidades, desejos e capacidades, proporcionando proteção, segurança e cuidados adequados. É necessário considerar o bem-estar físico, social e mental do idoso para que se tenha um envelhecimento ativo e participativo na sociedade em que está inserido. ${ }^{4}$

Segundo Lawton, ${ }^{5}$ qualidade de vida na velhice é uma avaliação multidimensional, referenciada a critérios socionormativos e intrapessoais, a respeito das relações atuais, passadas e prospectivas, entre o individuo maduro ou idoso e o seu ambiente. São variáveis relevantes na qualidade de vida de pessoas idosas o bem-estar psicológico, a qualidade de vida percebida, competência comportamental e as condições ambientais. Em seu estudo, Trentini ${ }^{6}$ relata associação entre maior intensidade de sintomas depressivos e pior qualidade de vida, e a percepção subjetiva de doença exercendo forte impacto na avaliação de qualidade de vida dos idosos.

Existe uma relação direta entre envelhecimento, qualidade de vida e religiosidade entre a população idosa. O termo "religiosidade" diz respeito ao nível de envolvimento religioso de um indivíduo e o quanto esse envolvimento influencia os seus hábitos cotidianos. ${ }^{7} \mathrm{~A}$ religiosidade é um sistema organizado de crenças, práticas, rituais de adoração, de doutrina e símbolos delineados para facilitar a proximidade com o sagrado e o transcendente de forma específica partilhada com um grupo. ${ }^{6}$ É um caminho para o relacionamento com algo ou alguém maior do que o mundo físico, e pode ser dividida em intrínseca e extrínseca. ${ }^{8}$

O tipo intrínseco predomina em indivíduos que demonstram um compromisso com a tradição de fé por razões sinceras que estão relacionadas com a busca de Deus, da verdade e do espírito 
de confraternização. Pessoas que possuem religiosidade intrínseca têm crenças internalizadas e encontram sua razão de vida na religião.

No tipo extrínseco, o indivíduo usa a religião para obter outros fins e/ou resolver interesses particulares. As crenças são levemente modificadas para encaixar em necessidades mais primárias, como proporcionar segurança, consolo, sociabilidade, distração, status e autoabsolvição. ${ }^{9}$

Medir a religiosidade intrínseca e extrínseca separadamente é extremamente difícil, já que todas as pessoas possuem características das duas feições, porém uma delas tende a imperar, o que identificará o indivíduo como tendo religiosidade intrínseca ou extrínseca. Também não podemos dizer que um indivíduo é melhor ou mais evoluído por ter a religiosidade intrínseca e o outro pior ou deficitário, por ter a religiosidade extrínseca, já que o ser humano está em constante mutação e muitos fatores podem influenciar direta ou indiretamente sua religiosidade. ${ }^{10}$

Por exercer a religiosidade um papel significativo frente aos desafios cotidianos geradores de estresse, propiciando maiores condições ao indivíduo idoso de lidar com a dependência e a tendência ao isolamento, o objetivo deste estudo foi avaliar a influência da orientação religiosa na qualidade de vida de idosos ativos.

\section{METODOLOGIA}

O estudo, de abordagem qualitativa, ocorreu na cidade de São José dos Campos (SP), junto aos idosos que frequentam o Centro de Atividades para Terceira Idade (Cativa), entidade sem fins lucrativos em funcionamento desde 1995, e o Centro de Referência Casa do Idoso, instituição municipal em funcionamento desde 2007. A população foi escolhida em função de os idosos participarem de diversas atividades nessas instituições (esportes, recreação, artesanato, leitura) sendo, portanto, considerados idosos ativos. Os dados foram coletados nas dependências das instituições, em dias em que os idosos já estavam presentes para realizar alguma atividade.

A pesquisa ocorreu no período de novembro de 2010 a fevereiro de 2011 e foram considerados elegíveis idosos com 60 anos e mais, de ambos os sexos, que participavam ao menos uma vez por semana de qualquer atividade (física, intelectual ou recreacional) em qualquer das duas instituições.

Todos os idosos, após a apresentação do projeto e esclarecimento de dúvidas, assinaram o termo de consentimento livre e esclarecido. $\mathrm{O}$ estudo foi conduzido de acordo com a Resolução n 196 , de 10 de outubro de 1996, do Conselho Nacional de Saúde do Ministério de Saúde (Brasília-DF), e aprovado pelo Comitê de Ética em Pesquisa da Universidade do Vale do Paraíba (UNIVAP), sob parecer n H137/CEP/2010. As recusas também foram registradas.

Em seguida, foi aplicado o teste cognitivo Mini-Mental State Exam (Mini-Exame do Estado Mental - MEEM), para garantir a integridade cognitiva dos participantes da pesquisa. ${ }^{11} \mathrm{O}$ MEEM é um teste composto por 11 itens, totalizando 30 pontos, abordando questões relacionadas à orientação, capacidade de registro, atenção, cálculo, memória e linguagem. O escore do MEEM pode variar de um mínimo de zero ponto, que indica maior grau de comprometimento cognitivo dos indivíduos, até um máximo de 30 pontos, que, por sua vez, correspondem à melhor capacidade cognitiva. Neste trabalho, foram incluídos apenas os idosos que apresentaram escore superior a 18 pontos. ${ }^{12}$

Dos 70 idosos convidados a participar da pesquisa, dez foram excluídos por motivos diferenciados (escore inferior a 18 pontos no MEEM, recusa por perceber que havia necessidade de escrever, falta de motivação para responder às perguntas e pouco tempo livre entre uma atividade e outra), compondo-se a amostra, então, por 60 indivíduos. Dentre estes, a média de idade foi de 73 anos (61 a 85 anos), sendo a maioria do sexo feminino $(82 \%)$. 
Em seguida, as pesquisadoras aplicaram um questionário sociodemográfico para caracterização da amostra, contendo as seguintes variáveis: sexo, idade, estado civil, escolaridade, cor, ocupação atual, renda salarial, arranjo familiar, moradia, hábitos e vícios, medicamentos em uso, comorbidades, ocorrência de quedas no último ano, análise subjetiva da visão e audição, além de questões relacionadas à prática de exercícios físicos e lazer e práticas como etilismo e tabagismo.

Com a finalidade de avaliar como as pessoas demonstram sua religiosidade, independentemente de credos específicos, foi utilizada a versão likert da Escala de Motivação Religiosa Intrínseca e Extrínseca. ${ }^{13}$ Esta é composta de 12 questões, traduzidas e adaptadas para o português por Goldstein, em 1993. ${ }^{14} \mathrm{O}$ escore total da escala pode variar de 12 a 60 pontos e, para se avaliar qual o tipo predominante de religiosidade, calcula-se a mediana dos dados.

Neste estudo, reproduzindo o processo realizado por Hoge, em 1972, as medianas, calculadas para cada indivíduo, foram colocadas em ordem crescente de valores. Desta série de dados, calculou-se a mediana da amostra estudada e comparou-se a mediana de cada indivíduo com a mediana da amostra. Foi considerado idoso com religiosidade intrínseca aquele cujo valor da mediana foi menor que o valor da mediana da população, e idoso com religiosidade extrínseca aquele cujo valor da mediana foi maior que o valor da mediana da população. ${ }^{15}$
Para avaliar a qualidade de vida dos idosos, foi utilizado o Medical Outcomes Short-Form Health Survey (SF-36), ${ }^{16}$ um instrumento de avaliação genérica da saúde, de fácil administração e compreensão para a população idosa. É constituído por 36 questões, que englobam oito escalas ou componentes para avaliação da capacidade funcional, aspectos físicos, dor, estado geral da saúde, vitalidade, aspectos sociais, aspectos emocionais, saúde mental e percepção subjetiva atual da saúde comparada há um ano. $\mathrm{O}$ indivíduo recebe um escore em cada domínio, podendo variar de zero (pior qualidade de vida) a 100 (melhor qualidade de vida). ${ }^{16}$

Para análise dos dados, foram realizados tratamentos estatísticos utilizando o teste $\mathrm{t}$ Student bilateral, a um nível de confiança de $5 \%$. As correlações amostrais foram testadas utilizando-se o teste $\mathrm{t}$ Student bilateral para significância das correlações. ${ }^{17}$

\section{RESULTADOS}

Em relação à naturalidade, $63 \%$ dos idosos pesquisados eram do Estado de São Paulo, $32 \%$ de outros Estados e 5\% declararam ter nascido em outros países. E quanto à etnia, 68\% declararam-se brancos, 28\% pardos ou negros e $3 \%$ amarelos. Dos pesquisados, apenas 38,3\% declararam morar sós. Perguntados sobre o uso de medicação apenas $6,7 \%$ relataram não fazer uso de nenhum medicamento. $O$ perfil sociodemográfico da amostra analisada está demonstrado na tabela 1 . 
Tabela 1 - Perfil sociodemográfico dos idosos estudados, de acordo com o tipo de religiosidade (em \%). São José dos Campos, SP - 2010/2011.

\begin{tabular}{llll}
\hline \multicolumn{1}{c}{ Variáveis } & RI & RM & RE \\
\hline Sexo feminino (n=49) & 26 & 35 & 39 \\
Casados (n=17) & 24 & 35 & 41 \\
Moram em casa própria (n=38) & 23 & 37 & 40 \\
Moram sozinhos (n=18) & 33 & 39 & 28 \\
Aposentados (n=41) & 34 & 46 & 20 \\
Atividade física regular (n=41) & 34 & 27 & 39 \\
Incontinência urinária (n=06) & 83 & 17 & -- \\
Incontinência fecal (n=03) & 100 & -- & -- \\
Etilistas (n=11) & 45 & -- & 55 \\
Tabagistas (n=04) & -- & -- & 100 \\
Quedas no último ano (n=14) & 14 & 29 & 57 \\
Analfabetos (n=03) & 34 & 33 & 33 \\
Renda pessoal de 1 a 3 SM (n=27) & 30 & 37 & 33 \\
Hipertensão arterial (n=32) & 25 & 31 & 44 \\
Osteoporose (n=20) & 28 & 34 & 38 \\
Depressão (n=15) & 26 & 37 & 37 \\
Artrose/artrite (n=16) & 30 & 35 & 35 \\
\hline
\end{tabular}

SM: salário mínimo (vigente $\mathrm{R} \$ 545,00) ; \mathrm{RI}=$ religiosidade intrínseca; $\mathrm{RM}=$ religiosidade mediana; $\mathrm{RE}=$ religiosidade extrínseca $* \mathrm{p}<0,05$ em relação às diferenças intra-grupo de cada variável analisada.

No questionamento sobre a religiosidade, 98\% declararam-se religiosos, sendo a maioria de católicos (81\%). Dentre a minoria, encontraramse idosos evangélicos (8\%), espíritas (3\%), além de outras religiões $(8 \%)$.

Quanto ao tipo de religiosidade, segundo a Escala de Motivação Religiosa Intrínseca e
Extrínseca, ${ }^{13} 23$ idosos (38\%) formaram o grupo daqueles com religiosidade extrínseca, e 18 (30\%) formaram o grupo daqueles com religiosidade intrínseca, enquanto constatou-se religiosidade mediana em 19 idosos (32\%). A distribuição por faixa etária e sexo, de acordo com o tipo de religiosidade, está demonstrada na figura 1. 


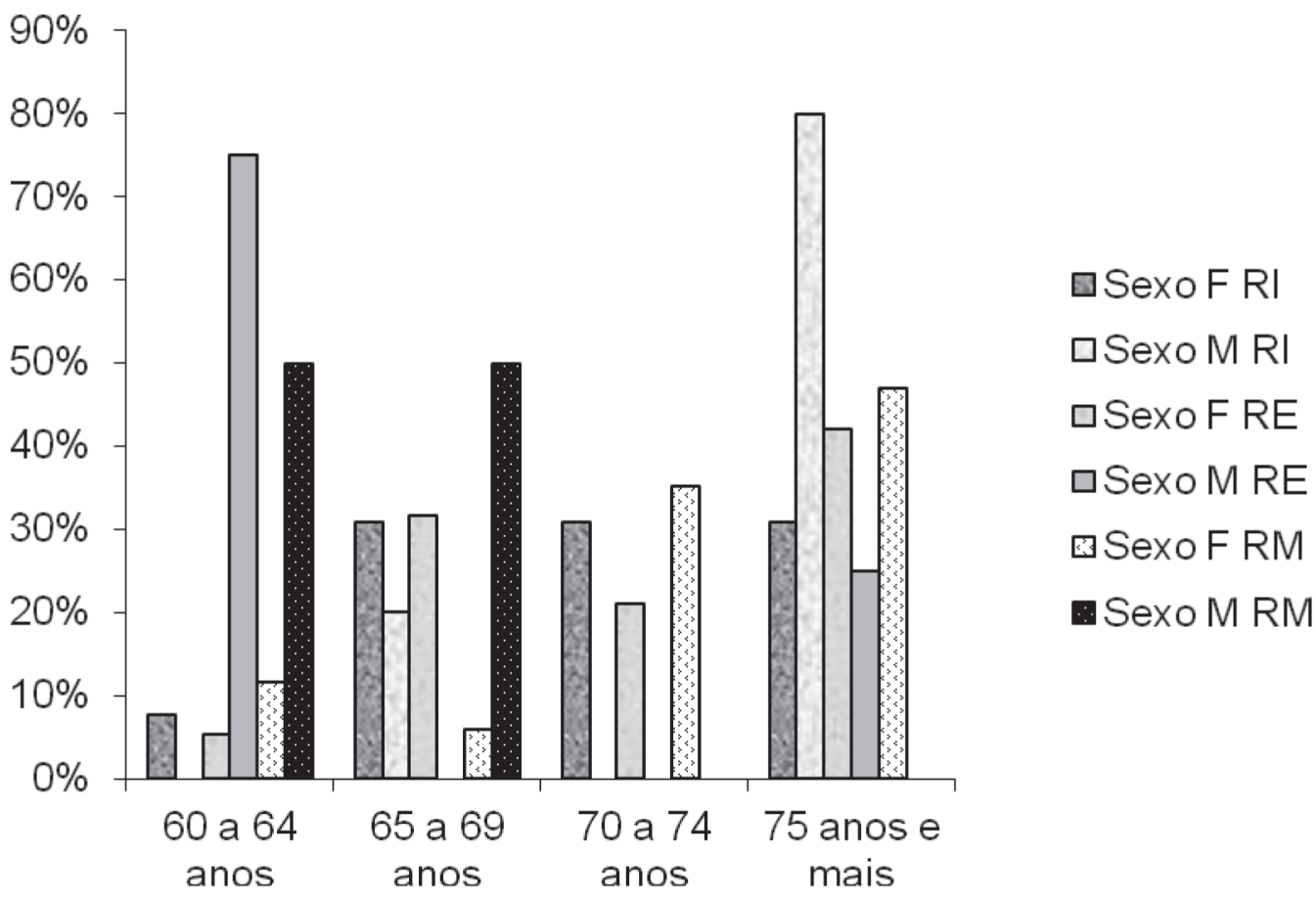

Sexo $\mathrm{F}=$ sexo feminino; Sexo $\mathrm{M}=$ sexo masculino; $\mathrm{RE}=$ religiosidade extrínseca $\mathrm{RI}=$ religiosidade intrínseca; $\mathrm{RM}=$ religiosidade mediana. * $\mathrm{p}<0,05$ em relação às diferenças intra-grupo de cada variável analisada.

Figura 1 - Distribuição da faixa etária da população idosa estudada em relação ao tipo de religiosidade e de acordo com o sexo (em \%) - São José dos Campos - SP - 2010/2011.

Com relação ao teste $\mathrm{SF}-36$, os três grupos analisados (religiosidade intrínseca, extrínseca e mediana) apresentaram-se satisfeitos com a qualidade de vida nos oito domínios analisados, com resultados acima de 58 pontos num total de 100, mas só foram encontradas diferenças estatisticamente significativas $(p=0,0074)$ no domínio "estado geral de saúde" entre homens com religiosidade extrínseca (67 pontos) e religiosidade intrínseca (95 pontos), conforme demonstrado nas figuras 2 e 3 . 


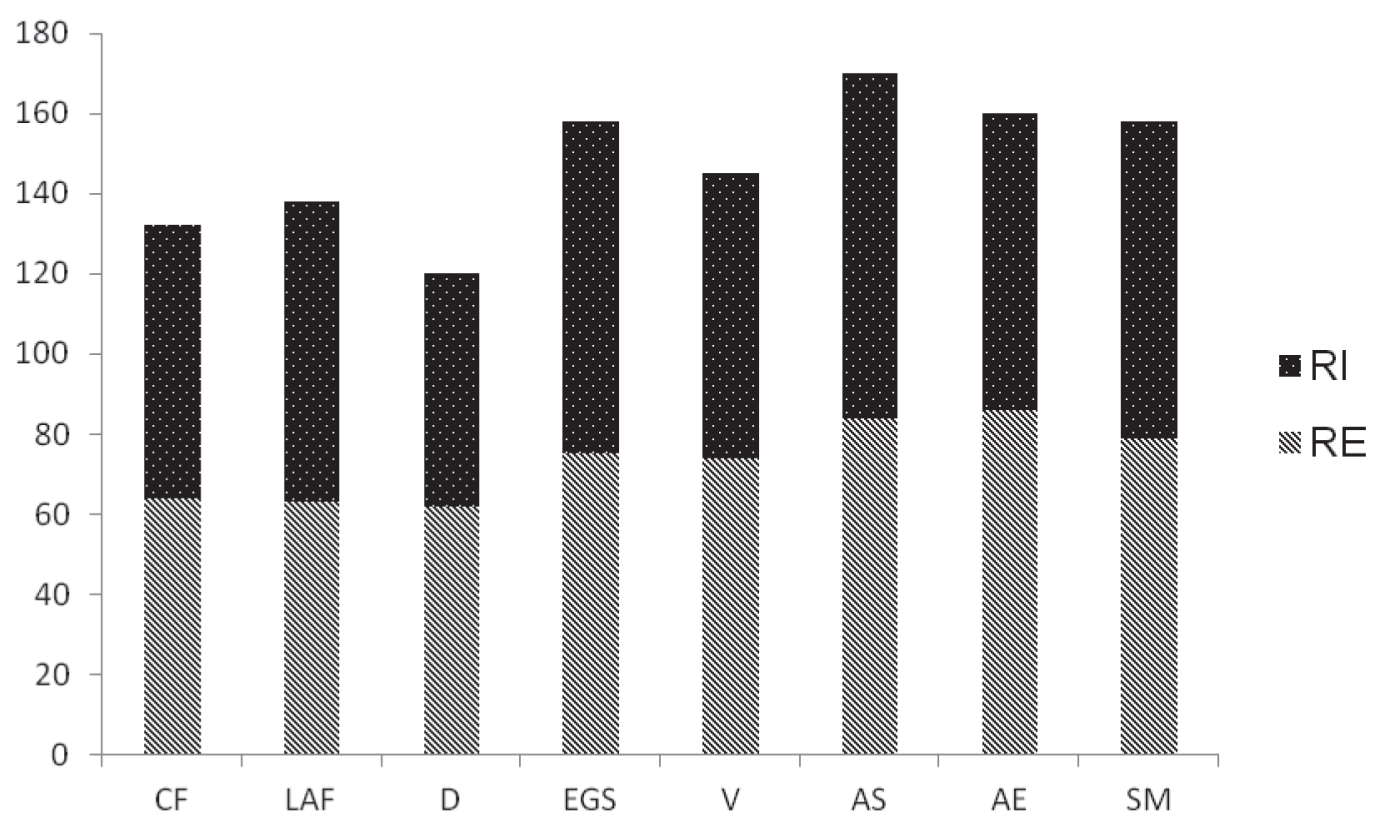

$\mathrm{RI}=$ religiosidade intrínseca; $\mathrm{RE}=$ religiosidade extrínseca $; \mathrm{CF}=$ capacidade funcional; $\mathrm{LAF}=$ limitação por aspectos físicos; $\mathrm{D}=$ dor; $\mathrm{EGS}$ = estado geral de saúde; $\mathrm{V}=$ vitalidade; $\mathrm{AS}$ = aspectos sociais; $\mathrm{AE}$ - aspecto emocional; $\mathrm{SM}$ = saúde mental.

$* \mathrm{p}<0,05$ em relação às diferenças intra-grupo de cada variável analisada

Figura 2 - Distribuição dos idosos do sexo feminino segundo a qualidade de vida (teste SF-36) em relação à religiosidade intrínseca (RI) e extrínseca (RE) (em \%) - São José dos Campos - SP - 2010/2011.

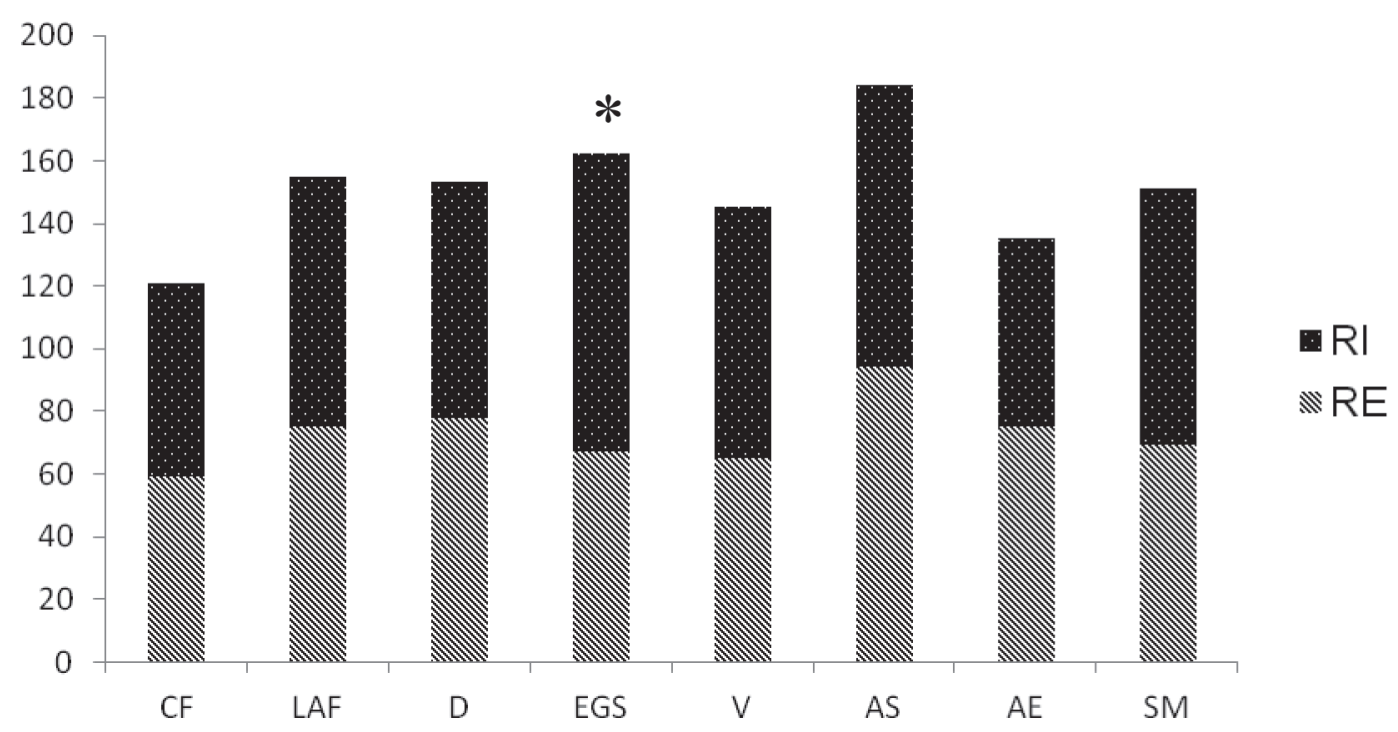

$\mathrm{RI}=$ religiosidade intrínseca $\mathrm{RE}=$ religiosidade extrínseca $; \mathrm{CF}=$ capacidade funcional; $\mathrm{LAF}=$ limitação por aspectos físicos; $\mathrm{D}=$ dor; $\mathrm{EGS}$ = estado geral de saúde; $\mathrm{V}=$ vitalidade; $\mathrm{AS}$ = aspectos sociais; $\mathrm{AE}$ - aspecto emocional; $\mathrm{SM}$ = saúde mental.

$* \mathrm{p}<0,05$ em relação às diferenças intragrupo de cada variável analisada

Figura 3 - Distribuição dos idosos do sexo masculino segundo a qualidade de vida (teste SF-36) em relação à religiosidade intrínseca (RI) e extrínseca (RE) (em \%) - São José dos Campos - SP - 2010/2011. 


\section{DISCUSSÃO}

Conforme se verificou nesta pesquisa, dos 60 idosos entrevistados, houve um predomínio do sexo feminino $(82 \%)$, o que vai ao encontro dos dados da SEAD (Secretaria Estadual de Assistência e Desenvolvimento Social do Governo do Estado de São Paulo), ${ }^{18,19}$ já que mulheres constituem a maioria da população nesta faixa etária no município de São José dos Campos. A faixa etária predominante na pesquisa realizada é de 75 anos ou mais, representando $40 \%$ da população estudada, sendo que no município a maior prevalência é da faixa etária de 60 a 65 anos. $^{19}$

De acordo com o arranjo familiar, a maioria dos idosos de nosso estudo reside com a família, geralmente com cônjuges e/ou filhos (58\%). Segundo dados do IBGE, ${ }^{20}$ esses arranjos familiares oferecem suporte familiar e estimulam a afetividade e sociabilidade, além de fornecer uma opção sociocultural que de certa forma compensa o poder socioeconômico. Além disso, 33\% dos idosos brasileiros moram com filhos, sendo este índice aumentado ao se falar em mulheres idosas (47\%). Já de acordo com o Projeto Epidoso, ${ }^{21}$ realizado com idosos do Estado de São Paulo, 90\% dos indivíduos residem em domicílios multigeracionais.

$\mathrm{Na}$ pesquisa realizada, as mulheres classificadas como de religiosidade extrínseca (47\%) não estão aposentadas e exercem atividades remuneradas. Quanto à renda pessoal, homens com religiosidade extrínseca e intrínseca declararam receber de três a cinco salários mínimos, o que é superior à renda das mulheres, que declararam ser de um a três salários mínimos.

No Estado de São Paulo, segundo o IBGE, ${ }^{20}$ $24,5 \%$ dos idosos nunca tiveram acesso à educação formal. Na amostra estudada, aproximadamente $7 \%$ declararam-se sem instrução e $37 \%$ possuíam de um a três anos de estudo, o que representa um nível de escolaridade básica inferior à média da região, que é de $20 \%$. No que diz respeito a nove anos ou mais de estudo, houve $32 \%$ dos idosos, sendo que o nível educacional supera o do Estado de São Paulo, que é de 20\%. De modo geral, os indicadores socioeconômicos da amostra de nosso estudo revelaram-se equiparados aos indicadores da população idosa do Estado.

Analisando-se a prática regular de atividade física dos frequentadores dos centros de convivência, constatou-se ser ela um hábito presente em 90\% dos idosos estudados, principalmente em mulheres com religiosidade intrínseca $(93 \%)$ e homens com religiosidade extrínseca $(100 \%)$. Em relação ao lazer, foram unânimes em afirmar que ir aos centros de convivência é a atividade que realizam com maior satisfação. Queiroz ${ }^{22}$ identificou que $90 \%$ das pessoas idosas de seu estudo consideraram que era importante conviver com pessoas da mesma faixa etária.

Os idosos participantes da pesquisa são ativos, têm autonomia, pouca ou nenhuma dependência, mas apresentam doenças comuns dessa fase de vida. No entanto, pouca relevância têm as comorbidades que apresentam quando realizam tratamento bem-sucedido, mantêm sua autonomia, sua capacidade funcional e estão integrados socialmente. ${ }^{21}$

Quanto ao requisito fundamental desta pesquisa, orientação religiosa, houve predominância da religiosidade extrínseca na população estudada, com 38,4\%, mas sem diferença estatisticamente significativa entre os tipos de religiosidade estudados. A escala aplicada foi considerada adequada à população idosa, por conter poucas questões e termos de fácil compreensão, que foram respondidas pelos entrevistados de acordo com a graduação que melhor expressasse seus sentimentos acerca dos aspectos religiosos, embora tenha havido necessidade de um despendimento maior de tempo para manter o rigor da pesquisa, o que para alguns idosos tornou-se cansativo.

A qualidade de vida dos idosos das instituições foi avaliada por meio do teste SF-36, pelos domínios: capacidade funcional (CF), limitação por aspectos físicos (LAF), dor (D), estado geral de saúde (EGS), vitalidade (V), aspectos sociais (AS), aspecto emocional (AE) e da saúde mental (SM). Após análise dos dados, evidenciou-se que 
os idosos associam uma boa qualidade de vida com a oportunidade de convivência social, a prática de atividades físicas, mentais e intelectuais e ao lazer, o que corrobora as informações da Organização Mundial da Saúde (OMS), ${ }^{3}$ de que os aspectos fundamentais para se viver com qualidade são as sensações íntimas de conforto, a percepção que o indivíduo tem de seu bem-estar físico, psíquico e social que somente ele pode avaliar e informar, livre de julgamento de valores externos a ele e que reflete os diversos aspectos da sua vida, dentro da realidade e dos valores da sociedade à qual pertence.

Ao se comparar os resultados obtidos no teste SF-36 com os obtidos na Escala de Orientação Religiosa, foi constatado que não houve diferença estatisticamente significativa nos oito domínios da escala para as idosas. Isto pode ser justificado devido ao fato de a mulher ser mais participativa, preocupar-se com as questões relacionadas ao bem-estar de um modo geral e estar em maior número nos grupos de convivência pesquisados, aumentando as possibilidades de se sentirem acolhidas e efetivarem laços de amizade, tendo sempre alguém que as escute. Além disso, tornam-se aumentadas as possibilidades de desenvolverem habilidades e potencialidades, antes adormecidas por suas responsabilidades familiares e domésticas, para o restabelecimento da autoimagem positiva, levando-as a ter uma percepção de autorrealização e bem-estar, parâmetros significativos na avaliação de qualidade de vida. ${ }^{23}$

Segundo Chacra,${ }^{24}$ qualquer atividade realizada em grupo, nas quais os integrantes possuam alguma identificação entre si, é uma atividade empreendedora de relacionamentos, estimulando o indivíduo a adquirir maior autonomia, melhorar sua autoestima, qualidade de vida, senso de humor e promover sua inclusão social.

Ao se comparar os resultados obtidos no teste SF-36 com os obtidos na Escala de Orientação Religiosa para os indivíduos do sexo masculino, não foram encontradas diferenças significativas do ponto de vista estatístico nos seguintes aspectos: capacidade funcional (CF), limitação por aspectos físicos (LAF), dor (D), vitalidade (V), aspectos sociais (AS), aspecto emocional (AE) e saúde mental (SM). Somente foram encontradas diferenças estatisticamente significativas no domínio "estado geral de saúde" (EGS), no qual homens com orientação religiosa intrínseca obtiveram escore próximo à pontuação máxima ( 95 pontos, $\mathrm{p}=0,0074$ ), o que pode ser justificado pelo fato de a religiosidade exercer papel significativo na existência humana, proporcionando um amparo seguro para lidar com os desafios cotidianos geradores de estresse, como isolamento, dependência, solidão e ansiedade, que permeiam a vida do idoso, muitas vezes ocasionando doenças. Assim, as crenças religiosas estimulam a adoção de práticas saudáveis por atribuírem significados aos fatos, favorecendo ao idoso a compreensão de que ele é parte de algo mais amplo. ${ }^{9,14,25,26}$

\section{CONCLUSÃO}

Numa abordagem mais ampla, pode-se argumentar que tantas semelhanças resultantes da correlação religiosidade e qualidade de vida se devem ao fato de a qualidade de vida ter um conceito multidimensional que envolve aspectos físicos, sociais e emocionais.

O tema da orientação religiosa não estranhava aos idosos, mas o resultado surpreendeu por não transparecer diferenças significativas entre as orientações religiosas dos tipos intrínseca e extrínseca. Esses resultados podem ter sido influenciados pelo fato de o município de São José dos Campos ter um índice de futuridade (ferramenta que mede a assistência prestada à pessoa idosa em termos de serviços, programas e iniciativas da gestão pública municipal) de $61 \%$, classificado como alto. Isto estaria garantindo a sua população idosa boas condições de atenção a suas necessidades de proteção, participação e saúde. Assim, devemos aventar que tais informações devem ser interpretadas com cautela, pois podem não ser representativas de outras regiões do país, com outras características sociodemográficas.

Algumas propostas para futuros estudos na área devem repensar o tempo de aplicabilidade 
dos instrumentos, visto que muitos idosos se sentiram cansados ao término dos quatro protocolos, além de tentar equiparar o mesmo número de homens e mulheres para as amostras.

Com estas considerações, espera-se que os resultados possam gerar subsídios para outras investigações sobre o tema.

\section{REFERÊNCIAS}

1. Maués CR, Paschoal SMP, Jaluul O, França CC, Filho WJ. Avaliação da qualidade de vida: comparação entre idosos jovens e muito idosos. Rev Bras Clin Med São Paulo 2010;8(5):405-10.

2. Pimenta FAP, Simil FF, Tôrres HOG, Amaral CFS, Rezende CF, Coelho TO et al. Avaliação da qualidade de vida de aposentados com a utilização do questionário SF-36. Rev Assoc Med Bras 2008;54(1):55-60.

3. Organização Mundial da Saúde. Divisão de Saúde Mental. Versão em português dos instrumentos de avaliação de qualidade de vida [WHOQOL] 1998. [acesso em 29 jul 2010]. Disponível em: http://www. ufrgs.br/psiq/whoqol1.html

4. Organização Pan-Americana da Saúde. Envelhecimento Ativo: uma Política de Saúde 2002. [acesso em 01 ago 2010]; Disponível em: http://bvsms.saude.gov.br/bvs/publicacoes/ envelhecimento_ativo.pdf

5. Lawton M. A multidimensional view of quality of life in frail elders. In: The Concept and Measurement of Quality of Life in the Frail Elderly. Birren J, Lubben J, Rowe J, Detchman D, eds. San Diego: Academic Press; 1991:3-27.

6. Trentini CM. Qualidade de vida em idosos. Porto Alegre. Tese [Doutorado em Ciências Médicas] Universidade Federal do Rio Grande do Sul; 2004.

7. Stroppa A, Moreira-Almeida A. Religiosidade e Saúde. In: Salgado MI, Freire G. Saúde e Espiritualidade: uma nova visão da medicina. Belo Horizonte: Inede; 2008.

8. Panzini RG, Rocha NS, Bandeira DR, Fleck MP. A. Qualidade de vida e espiritualidade. Rev Psiquiatr Clin 2007;34(1):105-15.

9. Araújo MFM, Almeida MI, Cidrack ML, Queiroz HMC, Pereira MCS, Menescal ZLC. O papel da religiosidade na promoção da saúde do idoso. Rev Bras Promoc Saúde 2008;21(3):201-08.

\section{AGRADECIMENTOS}

Aos idosos que aceitaram participar deste estudo. Aos dirigentes das instituições, que permitiram nossa presença em seus ambientes de trabalho. Em especial, ao engenheiro mecânico aeronáutico Reinaldo Shuhei Sakumoto, pelo auxílio prestado durante a análise de dados.

10. Araújo MIH. Orientação religiosa e qualidade de vida em idosos praticantes e não praticantes de exercícios físicos. Brasília. Dissertação [Mestrado em Gerontologia] - Universidade Católica de Brasília; 2005.

11. Folstein MF, Folstein SE, McHugh PR. "MiniMental State". A practical method for grading the cognitive state of patients for the clinician. J Psychiatr Res 1975;12(3):189-98.

12. Bertolucci PHF, Brucki SMD, Campacci SR, Juliano Y. O mini-exame do estado mental em uma população geral: impacto da escolaridade. Arq Neuropsiquiatr 1994;52(1):1-7.

13. Hoge R. A validated intrinsic religious motivation scale. J Sci Study Relig 1972;11(4): 369-76.

14. Goldstein LL. Desenvolvimento do adulto e religiosidade: uma questão de fé. In: Neri NA. Qualidade de vida e idade madura. Campinas: Papirus; 1993.

15. Drucker C. Religiosidade, crenças e atitudes em idosos deprimidos em um serviço de saúde mental de São Paulo, Brasil. Campinas. Dissertação [Mestrado em Gerontologia] - Universidade Estadual de Campinas; 2005.

16. Ciconelli RM, Ferraz MB, Santos W, Meinão I, Quaresma MR. Tradução para a língua Portuguesa e validação do questionário genérico de avaliação de qualidade de vida SF-36 (Brasil SF-36). Rev Bras Reumatol 1999;39(3):143-150.

17. Sampaio IBM. Estatística aplicada à experimentação animal. 2. ed. Belo Horizonte: FEPMVZ; 2002.

18. Governo do Estado de São Paulo. Secretaria Estadual de Assistência e Desenvolvimento Social. Plano Estadual para Pessoa Idosa (Futuridade) 2009. [acesso em 03 jun 2011]; Disponível em: http:// www.desenvolvimentosocial.sp.gov.br/usr/share/ documents/Plano $\% 20$ Estadual $\% 20$ para $\% 20 a \% 20$ Pessoa\%20Idosa.pdf 
19. Governo do Estado de São Paulo. Secretaria Estadual de Assistência e Desenvolvimento Social. Projeções Populacionais 2009. [acesso em 2011 jun 03]. Disponível em: http://www.seade.gov.br/ produtos/projpop/index.php

20. Instituto Brasileiro de Geografia Estatística. Síntese de Indicadores Sociais. Uma análise das condições de vida da população brasileira 2009. [acesso em 01 ago 2010]. Disponível em: http://www.ibge.gov. br/home/estatistica/populacao/condicaodevida/ indicadoresminimos/sinteseindicsociais2009/indic_ sociais2009.pdf

21. Ramos LR. Fatores determinantes do envelhecimento saudável em idosos residentes em centro urbano: Projeto Epidoso, São Paulo. Cad Saúde Pública, 2003;19(3):793-98.
22. Queiroz JB, Trinca SF. Influência do lazer sobre pessoas da terceira idade. Rev Bras Enf 1983;36(11):95-106.

23. Araújo LF, Coutinho MPL, Lucena e Carvalho VAM. Representações sociais da velhice entre idosos que participam de grupos de convivência. Psicol Cienc Prof 2005;25(1):118-131.

24. Chacra FC. Empatia e comunicação na relação médico-paciente: uma semiologia autopoiética do vínculo. Campinas. Tese [Doutorado em Saúde Coletiva] - Universidade Estadual de Campinas; 2002.

25. Oliveira TC, Araújo TL. Mecanismos desenvolvidos por idosos para enfrentar a hipertensão arterial. Rev Esc Enferm USP 2002;36(3):276-281.

26. Panzini RG, Bandeira DR. Escala de Coping Religioso-Espiritual (Escala CRE'1): elaboração e validação de um construto. Psicol Est 2005;10(3):507-16. 